\title{
Robust Matching of Wavelet Features for Sub-Pixel Registration of Landsat Data
}

\author{
Jacqueline Le Moigne \\ Applied Information \&Science \\ Branch \\ NASA/GSFC-Code 935 \\ Greenbelt, MD 20771 \\ Tel:301-286-8723 \\ Fax:301-286-1776 \\ lemoigne@backserv.gsfc.nasa.gov
}

\author{
Nathan S. Netanyahu \\ Dept Math. \& Comp.Sc. Bar-Ilan Univ., \\ Ramat-Gan, 52900, Israel, and Center \\ For Automation Research, Univ. of \\ Maryland, College Park, MD 20742 \\ nathan@cfar.umd.edu \\ David M. Mount \\ Computer Science Dept, University of \\ Maryland, College Park, MD 20742
}

\author{
Jeffrey G. Masek \\ Biospheric Sciences Branch \\ NASA/GSFC-Code 923 \\ Greenbelt, MD 20771 \\ jmasek@1tpmail.gsfc.nasa.gov
}

Samuel N. Goward

Geography Department, University of Maryland,

College Park, MD 20742

\begin{abstract}
For many Earth and Space Science applications, automatic geo-registration at sub-pixel accuracy has become a necessity. In this work, we are focusing on building an operational system, which will provide a sub-pixel accuracy registration of Landsat-5 and Landsat-7 data. The input to our registration method consists of scenes that have been geometrically and radiometrically corrected. Such preprocessed scenes are then geo-registered relative to a database of Landsat chips. The method assumes a transformation composed of a rotation and a translation, and utilizes rotationand translation-invariant wavelets to extract image features that are matched using statistically robust feature matching and a partial Hausdorff distance metric. The registration process is described and results on four Landsat input scenes of the Washington, D.C. area are presented.
\end{abstract}

\section{INTRODUCTION}

Over the next 30 years, NASA will be faced with many new challenges. In Earth Science, it will be the need to predict regional climate changes on seasonal and interannual time scales, or to understand the interactions between human activity and the changes in the major Earth ecosystems. In Space Science, distant planet exploration and formation flying will be part of many missions. To address such challenges, integrating and creating seamless mosaics of data from multiple times, multiple sensors, and multiple viewpoints will be a key component. Very accurate registration of these multi-sensor data is a fundamental prerequisite of such integration. But a number of distortions prevent two images acquired either by the same sensor at different times or by two sensors at the same or different times from being "perfectly registered" to each other or to a fixed coordinate system. It is very difficult to determine the exact location within an image using only ancillary data and geo-location is usually computed by combining navigation and registration. Navigation corresponds to a "systematic correction" based on image acquisition models taking into account satellite orbit and attitude, sensor characteristics, platform/sensor relationship, Earth surface and terrain models and brings the registration accuracy within a few pixels. Image registration, on the other hand, corresponds to a "precision correction" based on landmarks and image features, and refines the geo-location to sub-pixel accuracy. Registration is applied either after the navigation process, or both processes are integrated in a closed feedback loop. In this paper we will only consider the issue of feature-based, precision-correction automatic image registration.

Our goal is to build an operational system which will provide sub-pixel accuracy registration for Landsat-5 and Landsat-7 data. Our method assumes a transformation composed of a rotation and a translation, and utilizes rotation- and translation-invariant wavelets to extract image features [2,3], that are matched using statistically robust feature matching and a partial Hausdorff distance metric [4]. The registration is carried out on carefully chosen "subscenes" of a reference and of all incoming scenes. A preliminary report at IGARSS'00 [1] provided results of these individual sub-scene registrations. In this paper, we will first describe how these sub-scenes (or "chips" and "windows") are chosen and extracted. Then, we will summarize the principle of our algorithm, present new results, and show how results of all individual registrations are combined to provide the final registration of an input scene relative to a reference scene.

\section{WAVELET-BASED REGISTRATION OF LANDSAT DATA}

With the ultimate goal of integrating automatic registration within an automated mass processing/analysis system for Landsat data (REALM), we assume that the input to our registration method consists of scenes that are geometrically and radiometrically corrected. In the future, input scenes will also be pre-processed for detection of clouds and cloud shadows. Such pre-processed scenes are geo-registered by utilizing carefully chosen Landsat "reference chips," or "landmark chips." For the final system, a chip database will be built; we define "reference chips" as 256x256 images representing well-contrasted visual landmarks, such as bridges, city grids, islands, highcurvature points in coastlines. Several reference chips corresponding to different seasons and/or different reflectance conditions will represent each landmark area. For a typical Landsat input scene, we assume that between 5 and 10 well-distributed chips will be available from the database and will correspond to cloud-free areas of the scene. The choice of the relevant chips will be performed knowing the UTM (Universal Transverse Mercator) projection coordinates of one or the four corners of the scenes, and the 
UTM coordinates of all database chips. Currently, no chip database is available, so for the work presented in this paper, we created chips from a 1999 Landsat-7 scene, and then registered four Landsat- 5 scenes (acquired earlier) to those chips.

For each relevant reference chip that is selected as overlapping the given input scene, a corresponding window is extracted from the scene, using the UTM coordinates of the chip and of the scene corners. The UTM coordinates of the 4 chip corners are projected onto the scene with a simple linear interpolation taking into account the sizes (in meters) of the pixels, so that the projections are converted into pixel locations. These pixel locations are the basis for extracting a window in the scene that corresponds to the chip.

Then, each chip-window pair is now registered using our robust wavelet feature matching [4]. We assume that the transformation between incoming Landsat scenes and reference chips is limited to the composition of a rotation and a translation. Previous registration experiments [3] have shown that orthogonal wavelet filters could be utilized for image registration but were not rotation- and translationinvariant. Therefore such wavelet-based registration was not stable for large transformations and for large amount of noise. We then conducted similar experiments using the steerable filters from an overcomplete frame representation proposed by Simoncelli et al. [2], and the registration based on those new filters proved to be much more stable and more accurate than the previous one. For the registration of Landsat data, we decompose both chip and window using four decomposition levels and one steerable band-pass filter. At each level, the results of the band-pass filtering are thresholded to keep only the top $10 \%$ pixels whose magnitudes after filtering are the highest. Those top pixels at each decomposition level are used as features in the feature matching process.

The feature matching strategy follows the multiresolution given by the wavelet decomposition. Starting from the lowest level of decomposition and iteratively refining the matching at each level, strong features are matched using statistically robust feature matching and a partial Hausdorff distance metric. This matching is based on the principle of point mapping with feedback. Specifically, given a set of control points in the chip and a corresponding set of points in the scene window, and assuming a pre-specified transformation (here composition of translation and rotation), our method represents a computationally efficient algorithm to match these point patterns. An outline of our proposed algorithmic methodology consists of the following:

(0) Monte Carlo sampling of control points.

(1) Application of robust similarity measures (e.g., k-th smallest squared distance to nearest neighbor).

(2) Searching the transformation space through hierarchical spatial subdivisions.

(3) Pruning the search space by "range" similarity estimates.
(4) Employment of fast data structures for nearest neighbor and range queries in image space.

In summary, our registration algorithm can then be described in five steps:

1. For every new input scene, choose the relevant reference chips that have a sufficient overlap with the scene.

2. For each relevant reference chip, extract a corresponding window in the incoming scene.

3. For each chip-window pair, compute the (rotation, translation) transformation as follows:

3.1 Perform a wavelet decomposition of the chip and of the window. Extract the top $10 \%$ pixels whose wavelet magnitudes are the highest.

3.2 Perform a robust matching of the selected wavelet features using a nearest neighbor strategy and a partial Hausdorff distance.

4. A local transformation is computed for each chipwindow pair. From these local transformations, the corrected locations of the four corners of each chip are computed, and the list of all corners of all chips, including old and corrected locations, becomes the input of a robustified Least Mean Square (LMS) procedure that computes a global transformation over the entire input scene. If $\mathrm{n}$ is the number of chips that is used for the given scene, the global transformation is computed using $4 \mathrm{n}$ pairs of points.

5. Using the global transformation computed in step (4), new UTM coordinates for each of the four corners of the scene are being computed. This step provides a new scene indexing, and unless required by the user, no resampling is being performed.

\section{REGISTRATION RESULTS}

The method described in Section II was tested using seven 256x256 chips extracted from a 1999 Landsat-7 scene of the Washington/Baltimore area. Because the navigation system of Landsat-7 is more accurate than that of Landsat-5, we chose to utilize a 1999 Landsat-7 scene to extract the reference chips. Figure 1 shows these 7 chips. Four Landsat5 scenes from 1984, 1987, 1996 and 1997 over the same area were automatically registered to the chips. All scenes were projected using a WGS-84 model. Using the UTM coordinates of the 4 corners of each chip and the UTM coordinates of the 4 corners of the incoming Landsat-5 scene, a corresponding window was extracted for each chip. Figure 2 shows some of the corresponding windows. Then, each chip-window pair was automatically registered by extracting Simoncelli wavelet features up to level 4, and by matching them through robust feature matching. The results of all chip-window registrations are shown in Table 1, including the partial Hausdorff distance associated with each registration. Then, Table 2 shows the global registrations computed for all four scenes and Table 3 shows the corresponding registrations that were computed "manually" by averaging the locations of two Ground Control Points chosen visually. From these results, we can see that, 
quantitatively, the average translation error of the automatic registration compared to the manual registration is about 1 pixel in the $x$-direction and 0.63 pixel in the y-direction. The results also show that the translation errors are the smallest $(0.23,0.12)$ for scene 97275 , for which all distances associated with the local registrations are null, i.e. those registrations have very high confidence. This indicates that, if the confidence obtained in each local chip registration were taken into account when computing the global transformation, we could improve the final registration.

\section{CONCLUSION}

A system for the automatic registration of Landsat data has been designed. It was tested on four input Landsat-5 scenes registered to 7 chips extracted from a 1999 Landsat- 7 scene. Results are encouraging, but further testing needs to be performed, including introducing local registration confidence in the global transformation, as well as calculating the reference "ground truth" registration with a standard method such as ENVI. Future work will also include testing the method on a larger number of data, as well as building a well-distributed database of landmark chips.

\section{REFERENCES}

[1] J. Le Moigne et al., "Geo-registration of Landsat data by robust matching of wavelet features," IGARSS'2000.

[2] E. Simoncelli, and W. Freeman, "The steerable pyramid: a flexible architecture for multi-scale derivative computation," IEEE ICIP, October 1995.

[3] J. Le Moigne, and I. Zavorin, "Use of wavelets for image registration," SPIE/Aerosense'2000, Orlando, FL, Apr.00.

[4] D. Mount, N. Netanyahu, and J. Le Moigne, "Efficient algorithms for robust feature matching," Pattern Recognition, Vol.32, No.1, 1999.

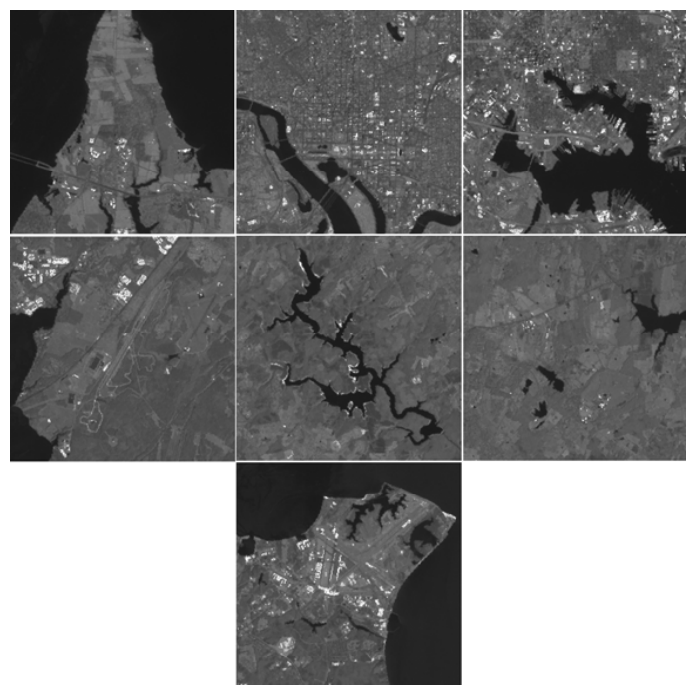

Figure 1

The 7 Chips Extracted from a 1999 Landsat-7 Band 4
Table 1- Local Chip-Window Pairs Transformations Rotations in degrees, Translations in pixels

\begin{tabular}{|l|l|l|l|l|}
\hline Chip \# & $\mathbf{8 4 2 4 0}$ & $\mathbf{8 7 1 3 6}$ & $\mathbf{9 6 1 9 3}$ & $\mathbf{9 7 2 7 5}$ \\
\hline \#1 - Rot: & 0 & 0 & 1 & 0 \\
TX & 8 & 18 & 12 & 21 \\
TY & -39 & -25 & -92 & -28 \\
Distance & 0.00 & 1.00 & 1.80 & 0.00 \\
\hline \#2 - Rot: & 0 & -0.5 & 1 & 0 \\
TX & 8 & 11 & -66 & 22 \\
TY & -41 & -41 & 4 & -30 \\
Distance & 0.00 & 0.62 & 1.93 & 0.00 \\
\hline \#3 - Rot: & 0 & -0.5 & -0.3 & 0 \\
TX & 8 & 11 & 10.84 & 21 \\
TY & -40 & -41 & -96 & -29 \\
Distance & 1.00 & 0.89 & 0.52 & 0.00 \\
\hline \#4 - Rot: & 0 & 0.8 & 0 & 0 \\
TX & 8 & 12.34 & 11 & 21 \\
TY & -41 & --38.12 & -94 & -28 \\
Distance & 1.00 & 0.96 & 0.00 & 0.00 \\
\hline \#5 - Rot: & 0.4 & 0.7 & 0 & 0 \\
TX & 7.8 & 10.4 & -38 & 20 \\
TY & -40.86 & --40.34 & 52 & -31 \\
Distance & 0.94 & 0.93 & 2.24 & 0.00 \\
\hline \#6 - Rot: & 0 & -0.8 & 0.3 & 0 \\
TX & 6 & 10.61 & 11.5 & 22 \\
TY & -41 & -41.94 & -99 & -33 \\
Distance & 1.00 & 0.78 & 0.70 & 0.00 \\
\hline \#7 - Rot: & 0.5 & 0 & 0 & 0 \\
TX & 9 & 12 & 12 & 22 \\
TY & -40 & -38 & -94 & -29 \\
Distance & 0.56 & 0.00 & 0.00 & 0.00 \\
\hline
\end{tabular}

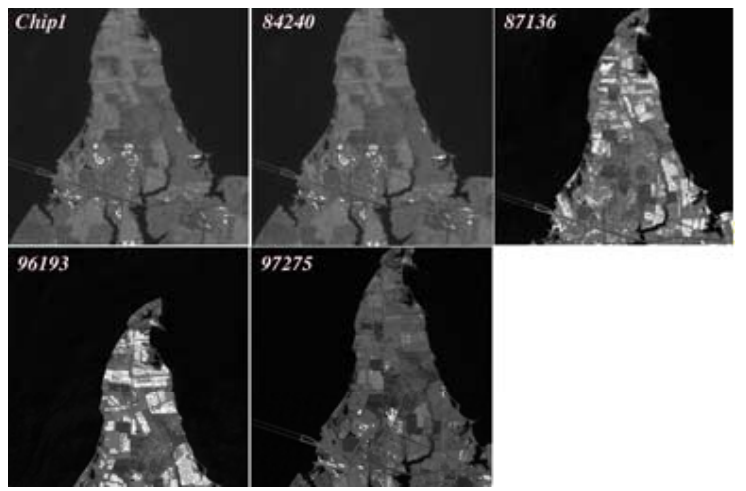

Figure 2

Chip1 and Its Corresponding Windows in the 4 Input Scenes

Table 2

Global Transformation For All Four Scenes

\begin{tabular}{|l|l|l|l|l|}
\hline Transf. & 84240 & 87136 & 96193 & 97275 \\
\hline Rotation & 0.013 & 0.003 & -0.042 & -0.143 \\
\hline Transl-x & 7.18 & 11.43 & 12.61 & 21.20 \\
\hline Transl-y & -41.12 & -40.49 & -95.38 & -28.85 \\
\hline
\end{tabular}

Table 3

Manual Transformation For All Four Scenes

\begin{tabular}{|l|l|l|l|l|}
\hline Transf. & 84240 & 87136 & 96193 & 97275 \\
\hline Rotation & 0.00 & 0.00 & 0.00 & 0.00 \\
\hline Transl-x & 7.18 & 10.55 & 9.48 & 20.97 \\
\hline Transl-y & -40.06 & -39.16 & -95.16 & -28.97 \\
\hline
\end{tabular}

